\title{
How reliable are bound-state parameters obtained from QCD sum rules?
}

\section{Wolfgang Lucha}

Institute for High Energy Physics, Austrian Academy of Sciences, Nikolsdorfergasse 18, A-1050,

Vienna, Austria

E-mail: Wolfgang.Lucha@oeaw.ac.at

\section{Dmitri Melikhov*}

Institute for High Energy Physics, Austrian Academy of Sciences, Nikolsdorfergasse 18, A-1050,

Vienna, Austria, and

D. V. Skobeltsyn Institute of Nuclear Physics, Moscow State University, 119991, Moscow, Russia

E-mail: dmitri_melikhov@gmx.de

\section{Silvano Simula}

INFN, Sezione di Roma III, Via della Vasca Navale 84, I-00146, Roma, Italy

E-mail: simulaeroma3.infn.it

\begin{abstract}
We discuss the extraction of ground-state parameters, such as decay constants and form factors, from two- and three-point dispersive sum rules, making use of a quantum-mechanical potential model. This model provides a unique possibility to probe the reliability and the accuracy of the method of sum rules: one obtains the bound-state parameters by the standard procedures adopted in sum rules, and compares these results with the exact values, known in the potential model. We demonstrate the presence of uncontrollable uncertainties in the ground-state parameters obtained from sum rules and estimate their magnitude. The uncontrolled uncertainties in ground-state form factors are shown to be typically much larger than those in the decay constants. In the example presented, the uncontrolled systematic error in the extracted form factor is found to exceed the $10 \%$ level.
\end{abstract}

8th Conference Quark Confinement and the Hadron Spectrum September 1-6, 2008

Mainz. Germany

\footnotetext{
*Speaker.
} 


\section{Introduction}

A QCD sum-rule calculation of hadron parameters [1,2] involves two steps: (i) one calculates the operator product expansion (OPE) for a relevant correlator and formulates the sum rule which relates this OPE to the sum over hadronic states, and (ii) one extracts ground-state parameters by a numerical procedure. Each of these steps leads to uncertainties in the final result.

The first step lies fully within QCD and allows for a rigorous treatment of the uncertainties: the correlator in QCD is not known precisely (because of uncertainties in quark masses, condensates, $\alpha_{s}$, radiative corrections, etc.) but the corresponding errors in the correlator may be controlled (at least in principle).

The second step lies beyond QCD and is more cumbersome: even if several terms of the OPE for the correlator were known precisely, the hadronic parameters may be extracted from a sum rule only with limited accuracy - the corresponding error has to be treated as a systematic error of the employed method.

In this talk, we present the results of our study of the systematic errors of hadron parameters obtained from dispersive sum rules. We address both the determination of the ground state's decay constant from the two-point correlator [3] and the extraction of the ground-state form factor from the three-point correlator [4] in a quantum-mechanical harmonic-oscillator (HO) potential model. This simple model has strong advantages compared to more complicated cases: (i) The bound-state parameters (masses, wave functions, form factors) are known precisely. (ii) One can calculate the exact two- and three-point functions. Thus, we may apply the standard sum-rule machinery to extract the ground-state parameters and compare them with the known exact values. In this way, we may probe the reliability and the accuracy of the method.

We shall demonstrate that the standard procedures adopted in the method of sum rules do not allow one to obtain rigorous error estimates for the ground-state characteristics. Moreover, we show that in the case of the form factors extracted from three-point correlators, the uncontrolled systematic errors may be considerably larger than those for the case of the decay constants extracted from two-point correlators.

\section{Harmonic-oscillator model}

We consider a nonrelativistic HO model defined by the Hamiltonian

$$
H=H_{0}+V(r), \quad H_{0}=\vec{p}^{2} / 2 m, \quad V(r)=m \omega^{2} r^{2} / 2, \quad r \equiv|\vec{r}| .
$$

In this HO model, all characteristics of the bound states are easily calculable. For instance, for the ground state $(n=0)$ one finds

$$
E_{0}=\frac{3}{2} \omega, \quad R_{0} \equiv\left|\Psi_{0}(\vec{r}=0)\right|^{2}=(m \omega / \pi)^{3 / 2}, \quad F_{0}(q)=\exp \left(-q^{2} / 4 m \omega\right),
$$

where the elastic form factor of the ground state is defined according to

$$
F_{0}(q)=\left\langle\Psi_{0}|J(\vec{q})| \Psi_{0}\right\rangle=\int d^{3} k \psi^{\dagger}(\vec{k}) \psi(\vec{k}-\vec{q}), \quad q \equiv|\vec{q}|,
$$

with the current operator $J(\vec{q})$ given by the kernel

$$
\left\langle\vec{r}^{\prime}|J(\vec{q})| \vec{r}\right\rangle=\exp (i \vec{q} \vec{r}) \delta^{(3)}\left(\vec{r}-\vec{r}^{\prime}\right) .
$$




\section{Polarization operator}

The basic quantity for the extraction of the ground-state wave function $\Psi_{0}$ at the origin (i.e., of the decay constant) in the method of dispersive sum rules is the correlator of two currents [1]. Its quantum-mechanical analogue is [5]

$$
\Pi(T)=\left\langle\vec{r}_{\mathrm{f}}=0|\exp (-H T)| \vec{r}_{\mathrm{i}}=0\right\rangle .
$$

For the HO potential, $\Pi(T)$ and its OPE (i.e., expansion at small Euclidean time $T$ ) are known [5]:

$$
\Pi(T)=\left(\frac{\omega m}{\pi}\right)^{3 / 2} \frac{1}{[2 \sinh (\omega T)]^{3 / 2}}=\left(\frac{m}{2 \pi T}\right)^{3 / 2}\left(1-\frac{1}{4} \omega^{2} T^{2}+\frac{19}{480} \omega^{4} T^{4}+\cdots\right) .
$$

\section{Vertex function}

The basic quantity for the extraction of the form factor in the method of dispersive sum rules is the correlator of three currents [2]. The analogue of this quantity in quantum mechanics is [4]

$$
\Gamma\left(\tau_{2}, \tau_{1}, q\right)=\left\langle\vec{r}_{\mathrm{f}}=0\left|\exp \left(-H \tau_{2}\right) J(\vec{q}) \exp \left(-H \tau_{1}\right)\right| \vec{r}_{\mathrm{i}}=0\right\rangle
$$

with the operator $J(\vec{q})$ given by (2.4). In the HO model, the exact analytic expression for $\Gamma\left(\tau_{2}, \tau_{1}, q\right)$ was obtained in [4]. The analogue of the OPE series, as used in the method of sum rules in QCD, takes for equal times $\tau_{1}=\tau_{2}=\frac{1}{2} T$ the following form:

$$
\begin{aligned}
& \Gamma_{\mathrm{OPE}}(T, q)=\Gamma_{0}(T, q)+\Gamma_{\text {power }}(T, q), \quad \Gamma_{0}(T, q)=\left(\frac{m}{2 \pi T}\right)^{3 / 2} \exp \left(-\frac{q^{2} T}{8 m}\right), \\
& \Gamma_{\text {power }}(T, q)=\left(\frac{m}{2 \pi T}\right)^{3 / 2}\left[-\frac{1}{4} \omega^{2} T^{2}+\frac{q^{2} \omega^{2}}{24 m} T^{3}+\left(\frac{19}{480} \omega^{4}-\frac{5 q^{4} \omega^{2}}{1536 m^{2}}\right) T^{4}+\cdots\right] .
\end{aligned}
$$

In our actual computations, we take into account terms up to $\mathrm{O}\left(T^{8}\right)$ and $\mathrm{O}\left(\omega^{6}\right)$ in $\Gamma_{\text {power }}$. Notice that the coefficients of each power of $T^{n}$ in the square brackets of (4.2) are polynomials in $q^{2}$. This feature restricts the applicability of three-point sum rules to the region of not too large $q^{2}$.

\section{Ground-state parameters}

Making use of the standard assumption that the ground-state contribution is dual to the lowenergy region of the free-quark diagrams, we obtain

$$
\begin{aligned}
R_{0} e^{-E_{0} T} & =\int_{0}^{z_{\text {eff }}(T)} d z \rho_{0}(z) e^{-z T}+\Pi_{\text {power }}(T), \\
R_{0} F_{0}(q) e^{-E_{0} T} & =\int_{0}^{z_{\text {eff }}(T, q)} d z_{1} \int_{0}^{z_{\mathrm{eff}}(T, q)} d z_{2} e^{-\frac{1}{2} z_{1} T} e^{-\frac{1}{2} z_{2} T} \Delta_{0}\left(z_{1}, z_{2}, q\right)+\Gamma_{\text {power }}(T, q) .
\end{aligned}
$$

Here $\rho_{0}(z)$ and $\Delta_{0}\left(z_{1}, z_{2}, q\right)$ are the known spectral densities of the two- and three-point Feynman diagrams of the nonrelativistic field theory [3, 4]. 
The relations (5.2) may be understood as the definition of the exact $T$-dependent (and, in the case of the form factor, also $q$-dependent) effective continuum thresholds, which are obtained as the solutions of Eqs. (5.2) for the exact bound-state parameters on the 1.h.s. The exact continuum thresholds (specific for each quantity) may be calculated in the HO model where these bound-state parameters are known, but cannot be obtained directly from the OPE. Obviously, the sum rules alone are not predictive: The ground-state parameters may be obtained in the method of sum rules only if one imposes an independent criterion to fix the effective continuum threshold.

Let us consider a restricted problem: namely, assume that the energy $E_{0}$ of the ground state is known and try to determine its decay constant and elastic form factor from the sum rules (5.2). The standard procedure adopted in this method is to approximate the effective continuum threshold by a $T$-independent quantity $z_{c}$ (for details, see [3, 4]). Following this line, we obtained the results depicted in Fig. 1. The parameter $R_{\mathrm{SR}}\left(T, z_{c}\right)$ extracted from the sum rule is very flat in the stability region but underestimates the known actual value by about $5 \%$. For the form factor, the situation is even worse: for instance, at $q_{0}=1.5 \omega$, the form factor $F_{\mathrm{SR}}\left(T, q_{0}, z_{c}\right)$ is perfectly flat in the stability region but, nevertheless, turns out to be more than $10 \%$ smaller than the known true value.

Let us emphasize the following dangerous point: (i) a perfect description of the correlators with an accuracy better than $1 \%$ in the stability region, (ii) the deviation of the energy from $E_{0}$ at the level of only $1 \%$, and (iii) a very good stability of the hadron parameters with better than $1 \%$ in the stability region lead, nevertheless, to an error of about $5 \%$ for $R_{0}$ and more than $10 \%$ in the extracted value of $F\left(q_{0}\right)$. Clearly, these errors could not be guessed on the basis of the other numbers found: the full picture mimics a very accurate extraction of the hadron parameters, which is, however, not true in reality!

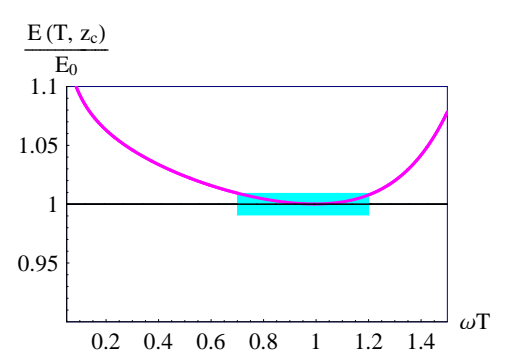

(a)

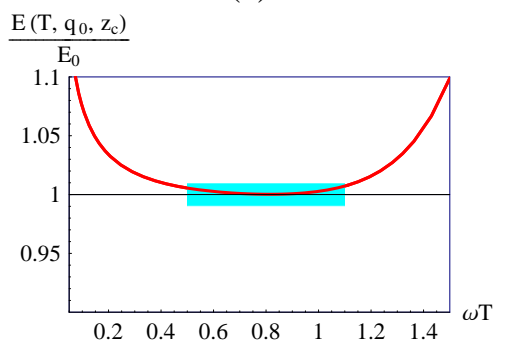

(c)

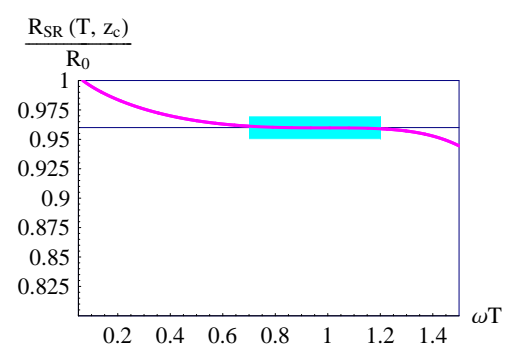

(b)

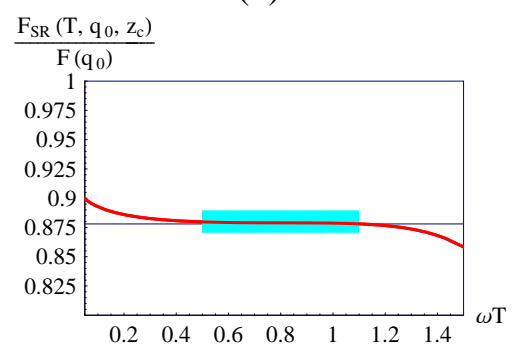

(d)

Figure 1: (a) The energy of the two-point cut correlator $E\left(T, z_{c}\right)=-\partial_{T} \log \Pi\left(T, z_{c}\right)$ (where $\partial_{T}$ is shorthand for $\partial_{T} \equiv \partial / \partial T$ ) and (b) the sum-rule estimate for the parameter $R_{0}$ for $z_{c}=2.454 \omega$. (c) The energy of the cut correlator $E\left(T, q_{0}, z_{c}\right)=-\partial_{T} \log \Gamma\left(T, q_{0}, z_{c}\right)$ and (d) the sum-rule estimate for the form factor at $q_{0}=1.5 \omega$ for $z_{c}=2.42 \omega$. The shaded rectangular areas indicate the regions of stability with $1 \%$ accuracy. 


\section{Summary and conclusions}

Let us summarize the lessons to be learnt from our analysis:

1. The knowledge of the correlator in a limited range of relatively small Euclidean times (equivalently, large Borel masses) is not sufficient for the extraction of the ground-state parameters. In addition to the OPE for the correlator, one needs an independent criterion for fixing the effective continuum threshold.

2. Assuming the effective continuum threshold to be Borel-parameter-independent allows one to fix this quantity by, e.g., requiring the average energy $E(T)$ to be close to $E_{0}$ in the stability region. In this case, however, the error of the extracted ground-state parameter turns out to be typically much larger than (i) the error of the description of the exact correlator by the truncated OPE and (ii) the variation of the bound-state parameter in the Borel window.

3. We stress that the stability of the extracted ground-state parameter in the Borel window - the standard criterion that is believed to control both the reliability and the accuracy of the extracted ground-state parameter - does not, in fact, guarantee the extraction of its true value.

4. The standard procedures for estimating the uncertainties of the extracted bound-state parameters do not allow one to provide realistic error estimates.

The impossibility to control the systematic errors of the extracted hadron parameters is the weak feature of the method of sum rules and an obstacle for using the results from dispersive sum rules in problems where rigorous error estimates are required.

Finally, we would like to comment on the obtained quantitative estimates. In the HO model, the ground state is well separated from the first excitation contributing to the correlator by the large gap of $2 \omega$. This feature renders the HO model a rather favourable case for the application of sum rules. Whether or not a comparable accuracy may be achieved in QCD — where the corresponding feature is absent - is questionable.

Acknowledgements. The authors would like to thank the organizers for arranging this very interesting meeting. D. M. gratefully acknowledges financial support from the Austrian Science Fund (FWF) under project P20573, and from the RFBR under project 07-02-00551.

\section{References}

[1] M. Shifman, A. Vainshtein, and V. Zakharov, QCD and resonance physics. Theoretical foundations, Nucl. Phys. B 147 (1979) 385.

[2] B. L. Ioffe and A. V. Smilga, Pion form factor at intermediate momentum transfer in QCD, Phys. Lett. B 114 (1982) 353; V. A. Nesterenko and A. V. Radyushkin, Sum rules and the pion form factor in QCD, Phys. Lett. B 115 (1982) 410.

[3] W. Lucha, D. Melikhov, and S. Simula, Systematic uncertainties of hadron parameters obtained with QCD sum rules, Phys. Rev. D 76 (2007) 036002; Can one control systematic errors of QCD sum-rule predictions for bound states? Phys. Lett. B 657 (2007) 148; Study of systematic errors of bound-state parameters in SVZ sum rules, Phys. Atom. Nucl. 71 (2008) 1461.

[4] W. Lucha, D. Melikhov, and S. Simula, Accuracy of bound-state form factors extracted from dispersive sum rules, arXiv:0810.1920 [hep-ph] .

[5] A. I. Vainshtein, V. I. Zakharov, V. A. Novikov, and M. A. Shifman, Asymptotic freedom in quantum mechanics, Yad. Fiz. 32 (1980) 1622. 